\title{
Performance Analysis of Double Gate Hetero Junction Tunnel Fet
}

\author{
Anjani Devi N, Ajaykumar Dharmireddy, Sreenivasa Rao Ijjada
}

\begin{abstract}
In this paper, a novel heterojunction tunnel field-effect transistor (HTFET) using Sentaurus technology computer-aided design (TCAD) simulations has been presented. The InAs/GaSb compound materials are used in both single gate heterojunction TFET (SG-HTFET) and Double gate heterojunction TFET (DG-HTFET) with SiO2 gate oxide layer to increase performance of the device.The implemented SG-HTFET and DG-HTFET device are increase the TFET's cross-sectional tunnel area. This result develops the subthreshold swing (SS) by 2.45 times, drive current $\left(I_{O N}\right)$ is close to $10^{-6} \mathrm{~A} / \mu \mathrm{m}$, leakage current $\left(I_{O F F}\right)$ is close to $10^{-17} \mathrm{~A} / \mu \mathrm{m}$ and also diminish the ambipolarity of the device compared to the TFET.
\end{abstract}

Keywords : Ambipolarity, Heterojunction TFET (HTFET), Double gate hetrojunction transistor (DG-HTFET), tunnelling field-effect transistor (TFET).

\section{INTRODUCTION}

One of the major challenge in the sub-30 nm regime's scaling of CMOS technology is power consumption[1]. A MOSFET's scaling under the 30-nm regime includes extreme supply voltage (VDD) scaling. Conversely, the subthreshold slope (SS) minimum fundamental limit on VDD scaling. To overcome this problem, energy filtering mechanisms-based devices such as TFETs are being more investigated to achieve the steep switching value below $60 \mathrm{mV} /$ decade(at room temperature) [2]. Using materials such as $\mathrm{Si}, \mathrm{Ge}, \mathrm{SiGe}$, and III-V materials several device structures have been implemented over the past decade to generate TFETs with high on current(Ion), low on/off current ratio (Ion/ Ioff)[3]-[5]. Because the band gap energy of InAs / GaSb compound materials is less, these materials are competitive among other material systems.

Due to their complete process technology, HTFET's constructed using III-V materials with limited lattice imbalance such as InAs / GaSb are of great attention to the system research community[6]-[8]. Area-scaled TFET systems have been developed in the recent past to improve the cross-sectional area of the device tunnelling [9],[10].The use of area-scaled TFETs increases the device footprint area compared to existing TFETs for the same channel length[5]. Therefore, to remedy this situation, a nonplanar architecture is required [11]. Recently, a tunnel junction structure developed by Hetro junction TFET (HTFET) was proposed

Revised Manuscript Received on December 13, 2019.

* Correspondence Author

Anjani Devi N *, Assistant Professor, Dept. of ECE, GRIET, Hyderabad, Telangana, INDIA. Email: anjali.nagineni@gmail.com

Ajaykumar Dharmireddy, Assistant Professor, Dept. of ECE, Sir CR Reddy college of Engineering, Eluru, AndhraPradesh, INDIA. Email: ajaybabuji@gmail.com

Sreenivasa Rao Ijjada, Assistant Professor, Dept. of EECE, GITAM srinivasanaidu.ijjada@gitam.edu Institute of Technology, Vishakhapatnam, Andhra Pradesh, INDIA. Email:

to increase the drive current with a reduced device footprint area[12].

The work is structured as follows: in section II the device structure and also the simulation setup for device optimization is elaborated. In section III, the proposed device simulations are presented. Comparison parameters in TFET, SG-HTFET and DG-HTFET in Section IV. And finally, the paper is concluded in Section V.

\section{DEVICE ARCHITECTURE AND SIMULATION SETUP}

On the source side, an HTFET structure is doped with InAs material and $\mathrm{GaSb}$ material as a channel and drain side doped material.

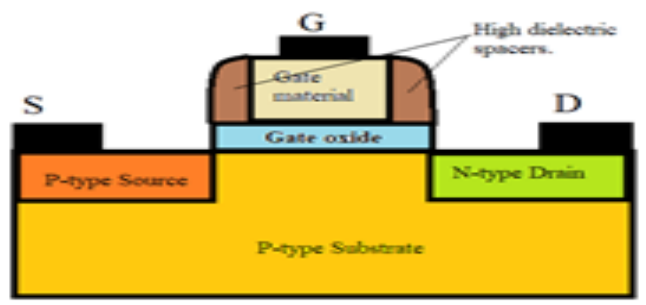

Fig. 1(a) single Gate HTFET.

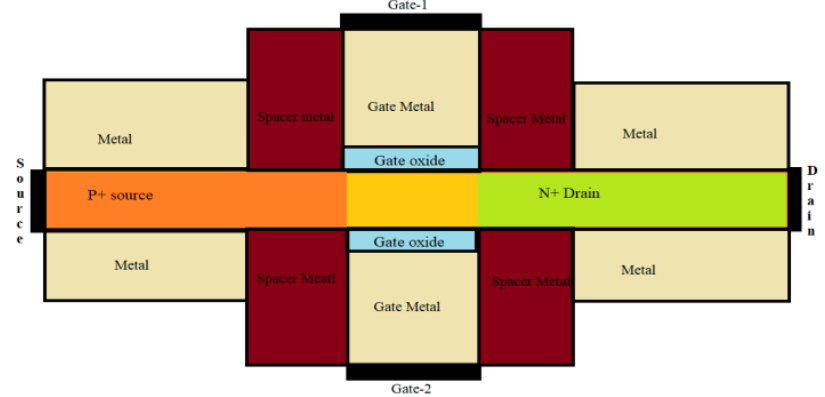

(b)Double gate HTFET

The SG-HTFET and DG-HTFET device cross section is shown in Fig. 1(a) and (b) above. Throughout the simulation, a metal gate work function $=4.6 \mathrm{eV}$ with a $3 \mathrm{~nm}$ gate oxide layer $(\mathrm{SiO} 2)(\mathrm{EOT}=0.5 \mathrm{~nm})$. The doping concentration of $5 \times 10^{19}$ is fixed for source and $5 \times 10^{18}$ for both drain and channel .

Table 1. Simulation setup of device parameters and values.

\begin{tabular}{|l|l|l|}
\hline S.NO & Para meters & values \\
\hline 1 & p-type doping (source) & $5 \times 10^{19} \mathrm{~cm}-3$ \\
\hline 2 & n-type doping (drain) & $5 \times 10^{18} \mathrm{~cm}-3$ \\
\hline 3 & n-type doping (channel) & $1 \times 10^{17} \mathrm{~cm}-3$ \\
\hline 4 & HfO2 material thick ness & $10 \mathrm{~nm}$ \\
\hline 5 & SiO2 material thick ness & $3 \mathrm{~nm}$ \\
\hline 6 & Gate metal thick ness & $7 \mathrm{~nm}$ \\
\hline 7 & Gate workfunction & $4.6 \mathrm{eV}$ \\
\hline
\end{tabular}

The geometric setup of the hetero junction TFET as InAS material is used in source, channel and substrate.

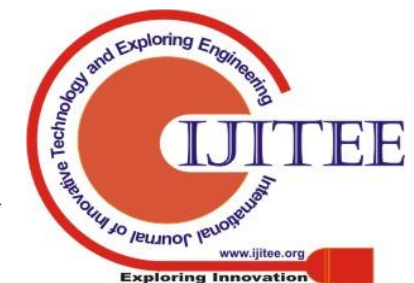


$\mathrm{GaSb}$ material is used to drain. HfO2 material is used in spacer and $\mathrm{SiO} 2$ material used in gate oxide .The nonlocal path band to band tunnelling model[13] was used to model the tunnel in the proposed structure. In accumulation, SRH recombination and band gap narrowing (old slot boom) models are used the device. For minimalism, quantum effects are not obviously constituted in the work except otherwise highlighted. The simulation setup of device parameters and values are presented in Table 1 [8], [14].

These devices get the same surface area, but in contrast with the TFET, the HTFET has a greater cross-sectional tunnel area. For a fair assessment, both devices ' epitaxial layer thickness $\left(\mathrm{t}_{\mathrm{CH}}\right)$ is set at $7 \mathrm{~nm}$ and channel length $\left(\mathrm{L}_{\mathrm{CH}}\right)$ at 20 nm.

\section{RESULTS AND DISCUSSION}

In fig. 2, $I_{D}$ versus $V_{G S}$ characteristics for HTFET is compared between drain current dynamic nonlocal path model and drain current dynamic barrier tunnel model. The dynamic barrier tunnel model performance is better than the dynamic nonlocal path model because the barrier height is changed with respect to the applied voltage The energy barrier in this condition is narrow enough where electrons will tunnel from the $\mathrm{p}+$ region's valance band to the intrinsic region's conductive band. The non-local band-to-band tunnelling through the tunnel barrier and the overall bi-polar nature of the current flow are evident.

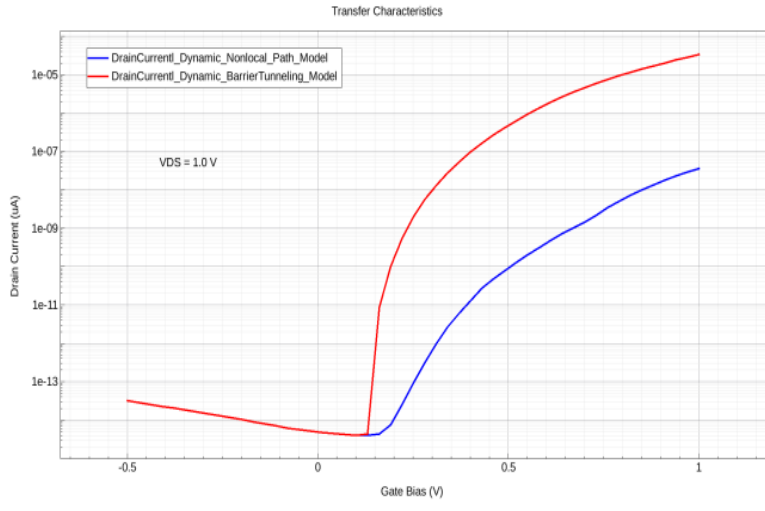

Figure 2. $I_{D}$ vs $V_{G S}$ characteristics comparison for DG-HTFET

Also note that current flow and tunneling are fairly evenly distributed across the channel width, at least in this thin-fin DG structure, even without consideration of quantum effects; the device operates in a near-flat-band condition.

For each device, many such simulations have been IDS-|VDS| characteristics. For initial |IDS|-VG simulations, the source VS and drain VD voltages for the n-channel single gate and double gate HTFET devices, were biased as if in the OFF-state or just switched to the ON-state within a CMOS-like inverter, although with a supply voltage VDD of only $75 \mathrm{mV}$. For the n-channel devices, the source was grounded and VD was set to VDD; All devices show very abrupt switching in the critical sub-threshold regime. performed to obtain and understand their |IDS|-VG and

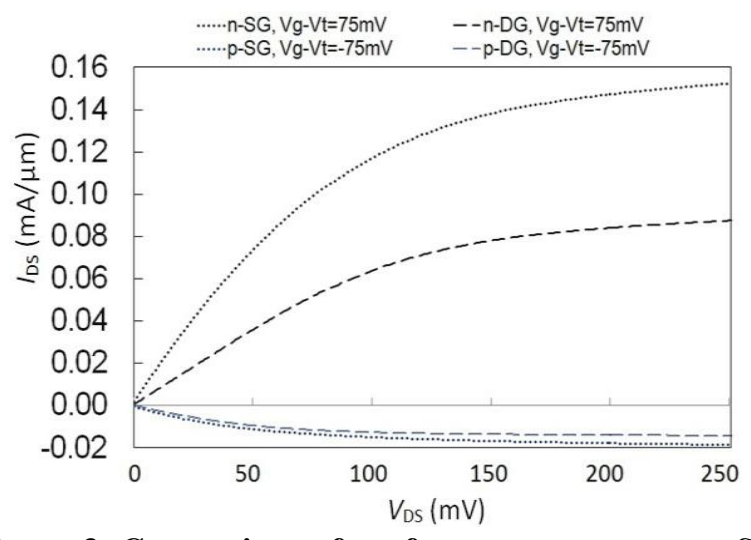

Figure 3. Comparison of performance parameters SG and DG-HTFET $I_{D S}$ versus $V_{D S}$ characteristics

Finally, Figure 3 shows the $I_{D S} v s .\left|V_{D}-V_{S}\right|$ for a $75 \mathrm{mV}$ gate overdrive voltage $\left|\mathrm{V}_{\mathrm{G}}-\mathrm{V}_{\mathrm{Th}}\right|$. As can be seen, saturation is more difficult to achieve at low voltages. For $\left|V_{D}-V_{S}\right|$ of only a few kBT, back injection of charge carriers from the source would prevent saturation. The lack of saturation beyond that, however suggest perhaps some effect of the drain voltage on the channel tunneling barrier and/or channel potential profile, the TFET equivalent of drain-induced-barrier lowering. Of more concern than the lack of $\mathrm{I}_{\mathrm{DS}}$ current saturation would be variation in the gate $\mathrm{V}_{T h}$ with $\left|\mathrm{V}_{\mathrm{D}}-\mathrm{V}_{\mathrm{S}}\right|$.

\section{Performance Comparison of DeVice PARAMETERS}

To get a superior perception into the performance of the SG-HTFET and DG-HTFET device, the device parameters of both HTFET devices are associated with conventional TFET in this module. The drain current $\left(\mathrm{I}_{\mathrm{D}}\right)$ versus $\mathrm{V}_{\mathrm{GS}}$ curves of both devices are shown in Fig.3. The both HTFET devices results are superior to the conventional TFET at the VGS and the VDS value of 1.0 and $0.5 \mathrm{~V}$, correspondingly.

Table 2. Lists of the work out electrical parameters of the DG-TFET

\begin{tabular}{|c|c|c|c|}
\hline PARAMETERS & TFET & SG-HTFET & DGTFE \\
\hline $\begin{array}{c}\text { SS } \\
(\mathbf{m V} / \mathbf{d e c a d e})\end{array}$ & 43.99 & 41.59 & 41.54 \\
\hline $\mathbf{I}_{\text {ON }}(\boldsymbol{\mu} \mathbf{A} / \boldsymbol{\mu m})$ & 43.8 & 5.26 & 5.84 \\
\hline $\mathbf{I}_{\text {OFF }}(\boldsymbol{\mu} \mathbf{A} / \boldsymbol{\mu m})$ & 40.3 & 25.2 & 24.3 \\
\hline $\mathbf{I}_{\text {ON }} / \mathbf{I}_{\text {OFF }}$ & $1.09 \times 1$ & $2.087 \times 10$ & $\begin{array}{c}2.403 \times 1 \\
0^{12}\end{array}$ \\
\hline
\end{tabular}

The enhancement in drive current of the DG-HTFET device is due to the better Sub-threshold slope value of the device that is attributed to the greater tunneling area and improved gate control in the overlap region.

It is evident from the results that, the drain current performance is improving with replacement of high-k gate materials instead of using conventional $\mathrm{SiO}_{2}$. Threshold voltage $\left(\mathrm{V}_{\mathrm{th}}\right)$ of DG-TFET reduces with high-k gate, as shown in Table 2 due to improved electrostatic filed inside tunneling region. This is also a scientific indication for lower power supply. 
The output characteristic is improved with increasing drain voltage and achieves its optimum value for drain voltage $V_{D S}$ equal to $1.0 \mathrm{~V}$. The use of high-k improves the output characteristics for a low gate voltage of the order of $0.5 \mathrm{~V}$. An increase in the drain voltage $\mathrm{V}_{\mathrm{DS}}$ greatly increased $\mathrm{I}_{\mathrm{ON}}$ current but had no effect on the threshold voltage.

\section{Conclusions}

Dynamic band-to-band tunnelling depends mainly upon barrier width and band alignment in III-V semiconductor based heterostructures and process materials for TFETs [15]. Improved control over the barrier shape can be obtained by strategically placing the hetero-interfaces, and desired band alignment can be achieved by changing the mole fraction of these compound materials. III-V materials also generally offer higher mobility, which further improves the subthreshold swing (SS) by 2.45 times, drive current $\left(\mathrm{I}_{\mathrm{ON}}\right)$ is close to $10^{-6} \mathrm{~A} / \mu \mathrm{m}$, leakage current $\left(\mathrm{I}_{\mathrm{OFF}}\right)$ is close to $10^{-17}$ $\mathrm{A} / \mu \mathrm{m}$ compared to the TFET. Besides, these structures of single gate HTFET and Double gate HTFET diminish the ambipolarity of the device in evaluation to the conventional TFET

\section{ACKNOWLEDGMENT}

It is optional. The preferred spelling of the word "acknowledgment" in American English is without an "e" after the "g." Use the singular heading even if you have many acknowledgments. Avoid expressions such as "One of us (S.B.A.) would like to thank ... ." Instead, write "F. A. Author thanks" Sponsor and financial support acknowledgments are placed in the unnumbered footnote on the first page.

\section{REFERENCES}

1. Aryan Afzalian,Gerben doorknobs,T Zer-Min Shen,Matthias Passlack. "A high performance InAs/GaSb Core-shell nanowire line tunneling TFET" An atomistic mode space NEGF study, Electron design society, 2019

2. 2008 update of the ITRS, http://www.itrs.net

3. Anjani Devi N, Ajaykumar Dharmireddy, Sreenivasa Rao Ijjada, "Performance Characteristics Of TFET Over MOSFET, DG-MOSFET and FinFET" International journal of innovative technology and exploring engineering (IJSAEE),pp187-193,2019.

4. Muhammad Elgamal and Mostafa Fedawy.: Optimizing Gate-on-Source Overlapped TFET Device Parameters by Changing Gate Differential Work Function and Overlap Dielectric, International Conference on Innovative Trends in Computer Engineering, February 2019.

5. D.Ajaykumar,I.srinivasarao,A.Ramkumar"A Perspective Review of Challenges on FinFET Technology in Low Power and High Speed Circuit Designs" Springer Lecture Notes in Electrical Engineering (LNE), 2019.

6. TCAD Taurus Device, http://www.synopsys.com

7. S.Satya Narayana, D.Ajay Kumar, Dr.Sreenivasarao Ijjada "Low Energy utilization of 14nm FINFET technology 6T-SRAM style" Journal of Emerging Technologies and Innovative Research (JETIR), Vol. 5, Issue 12,pp.112-117, December 2018.

8. David C, Francesc M, Stanimir V.: Prospects of Tunnel FET devices for Energy Harvesting Power Management Circuit design, Journal of Electronic device society, 2018.

9. Sreenivasa Rao I, Chaithanya M and Md. Hameed Pasha.: FinFET Modeling using TCAD, Springer Lecture Notes in Electrical Engineering (LNE), 2017

10. M Schmidt, A Schafer, R A Minamisawa, D Buca, S Trellenkamp, J M Hartmann, Q T Zhao and S Mantl.: Line and Point Tunneling in Scaled Si/SiGe Heterostructure TFETs, IEEE Electron Device Letters, JULY 2014.
11. M. Enz, "Indirect Electron Tunneling," [Online document], May 1998, Available: http://mp.physics.um.edu

12. Memisevic, Elvedin "Vertical III-V Nanowire Tunnel Field-Effect "Doctoral Thesis Electrical Engineering Lund, September 2017.

13. Mandeep Singh, Gaurav Kumar, Sushanta Bordoloi and Gaurav Trivedi "A study on modeling and simulation of MultipleGate MOSFET" Journal of Physics: Conference Series 759 (2016) 012093 doi:10.1088/1742-6596/759/1/012093

14. K.Shiromani Balmukund,Rahi1 - Pranav Asthana,Shoubhik Gupta. "Heterogate junctionless tunnel field-effect transistor: future of low-power devices" J Comput Electron (2017) 16:30-38 DOI 10.1007/s10825-016-0936-9

15. D. Shahrjerdi, D. Garcia-Guteirrez, S. Kim, M. M. Hasan, K. Varahramyan, E. Tutuc, and S. K. Banerjee, "Fabrication of Self-aligned Enhancementmode n-channel GaAs MOSFETs Employing a Wet Clean Process for GaAs Substrates," ECS Transactions, Vol. 16, Issue 4, p. 59 (2008).

\section{AUTHORS PROFILE}

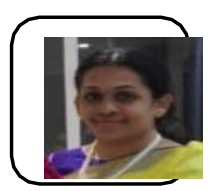

N. Anjani Devi received her B.E degree from SIR.C.R.Reddy College of Engineering affiliated to Andhra university in the year of 2005 and received M.Tech degree in the year of 2010 from the ASTRA, Hyderabad, Telangana affiliated to JNTUH University. She is pursuing Ph.D in GITAM Institute of Technology,

GITAM University, Visakhapatnam and presently working as an Assistant Professor in GRIET,Hyderabad,Telangana. Her research interests include Low power VLSI Design,FinFET technology

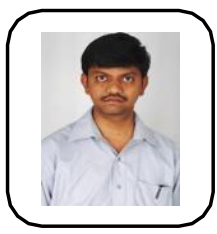

D.Ajay Kumar received his Btech degree from BVCIT\&S,Amalapuram affiliated to JNTUH in the year of 2006 and received M.E degree in the year of 2009 from the Bannari Amman Institute Of Technology, Sathyamagalam, Tamilnadu affiliated to ANNA University, Coimbatore. He is pursuing Ph.D in GITAM Institute of Technology, GITAMUniversity,

Visakhapatnam and presently working as an Assistant Professor in SIR.C.R.Reddy College of Engineering, Eluru,Andhra pradesh. His researchinterestsincludeLowpowerVLSIDesign,FinFETtechnology.He is a life member of the IEI ,ISTE andIAENG.

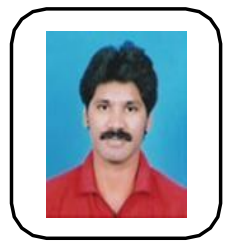

Dr.Srinivasa Rao.Ijjada received his AMIE degree from The Institution of Engineers (INDIA) in the year 2001 and received M.Tech degree in the year 2006 from JNTU Kakinada .He completed his Ph.D from GITAM University, Visakhapatnam and currently working as an Assistant Professor in GITAM Institute of Technology, GITAM University, Visakhapatnam,

Andhra pradesh. He is senior member IEEE, Research gate and life member of AMIE. He published several reputed research journals. His research activities are related to Low Power VLSI Design, FinFET technology,Microwave and Bio-Signal Processing. 